\title{
On Finite-Dimensional Transformations of Anisochronic Controllers Designed by Algebraic Means: A User Interface
}

\author{
Libor Pekař, Eva Kurečková and Roman Prokop \\ Additional information is available at the end of the chapter
}

http://dx.doi.org/10.5772/46472

\section{Introduction}

Linear time-invariant time delay systems (LTI TDS) have usually been assumed to contain delay elements in input-output relations only. All the system dynamics has been hence modeled by point accumulations in the form of a set of ordinary differential equations. The Laplace transform then results in a transfer function expressed by a serial combination of a delayless term and a delay element. However, this conception is somewhat restrictive in effort to fit the real plant dynamics because inner feedbacks are often of the time-distributed or delayed nature.

Anisochronic (or hereditary) TDS models, on the other hand, offer a more universal dynamics description applying both integrators and delay elements either in lumped or distributed form so that delays appear on the left side of a differential equation which is no longer ordinary (ODE) but rather functional (FDE) - this brings the concept of internal (or state) delays. In contrast to undelayed systems, the main difference in dynamics is that their spectra are infinite in general. In the further text, an abbreviation TDS means LTI TDS containing state delays with or without input-output delays.

Already in (Volterra, 1928) differential equations incorporating the past states when studying predator-pray models were formulated. The theory of these models has been then developed by many outstanding authors, see e.g. (Bellman \& Cooke, 1963), (Krasovskii, 1963), (Kolmanovskii \& Nosov, 1986), (Zítek, 1983), (Górecki et al., 1989), and especially (Hale \& Verduyn Lunel, 1993) and (Nicolescu, 2001), to name a few. Aftereffect phenomenon is included in many processes, e.g. in chemical processes (Zítek \& Hlava, 2001), heat exchange networks (Zítek, 1997), in models of mass flow in sugar factory (Findeisen et al., 1970), in metallurgic processes (Morávka \& Michálek, 2008), etc. Plenty of 
references to examples of processes with internal delays, covering a wide range of human activities (e.g. biology, chemistry, economics, communication and information technologies, etc.) are introduced in (Kolmanovskii \& Myshkis, 1999; Niculescu 2001; Richard, 2003). Capabilities and advantages of this class of models and controllers for modeling and process control were broadly discussed in (Manitius \& Olbrot, 1979). TDS models can be used not only for description of those systems embodying internal delays but they are successfully capable to fit the dynamics of high-order systems and processes even without apparent delays (Zítek \& Víteček, 1999; Vyhlídal \& Zítek, 2001; Simuenovic, 2011), which simplifies the processes description.

Using the Laplace transform applied to FDEs of TDS, input-output models in the singleinput single-output (SISO) case can be expressed by the transfer function in the form of a fraction of so-called quasipolynomials (El'sgol'ts \& Norkin, 1973) which can be viewed as polynomials in complex variable $s$ over the ring of exponentials polynomials (or over a ring of linear combinations of real numbers and exponential functions in $s$ ). The concept of pseudopolynomials (Brethé \& Loiseau, 1998), meromorphic functions (Zítek \& Kučera, 2003) or a special ring (Gluesing-Lueerssen, 1997; Conte \& Perdon, 2000), instead of quasipolynomials, can be used as alternatives; however, from the description point of view all these models are equivalent - their significance emerges while controller design.

Delay in the feedback control significantly deteriorates control performance, namely stability and periodicity, and makes controller design more complex - mainly if delays are not approximated or ignored. Therefore, design a suitable control law for such systems is a challenging task solved by various techniques and approaches; a plentiful enumeration of them can be found e.g. in (Richard, 2003). Due to the complexity of many direct methods, one can approximate an infinite-dimensional model by a finite-dimensional one to obtain a rational transfer function which can be handled by any standard control approach for undelayed plants. Similarly, in case of delayed (anisochronic) controllers obtained by controller design without delay approximation, one way how to implement these control laws on discrete-time-working machines such as PLC or PC is to find a delayless (i.e. finitedimensional) approximating model followed by a discretization (e.g. by the z-transform with a holder).

This chapter aims two problems. First, output controller design for TDS models in a special ring is introduced. Second, resulting anisochronic controllers are approximated using various techniques for rational approximation of transfer function of TDS, which is the crucial part of the chapter. Hence, let us to make a brief insight into the two tasks.

Some authors, e.g. in (Brethé \& Loiseau, 1998), pointed out that the use quasipolynomials does not permit to effectively handle some stabilization and control tasks, such as internal stability, controller properness etc. Hence, the quasipolynomial (meromorphic) description of TDS can be extended to a fractional one where a transfer function can be viewed as a field of fractions over a ring. The ring of stable and proper quasipolynomial (RQ) meromorphic functions (RMS) (Zítek \& Kučera, 2003; Pekař \& Prokop, 2010; Pekař \& Prokop, 2011a, 2011b; Pekař et al., 2011) is a suitable candidate. Although the ring can be used for a description of 
even neutral systems (Hale \& Verduyn Lunel, 1993) after some definition adjustment, only systems with so-called retarded structure are considered as the admissible class of systems in this paper. Contrary to some other algebraic approaches, the ring enables to handle systems with non-commensurate delays, i.e. it is not necessary that all system delays can be expressed as integer multiples of the smallest one. Control philosophy in this ring then utilizes the Bézout identity, to obtain stable and proper controllers, along with the YoulaKučera parameterization for reference tracking and load disturbance rejection.

Final controllers, in most cases, evince internal delays. Hence, a special effort to apply the control law in discrete time when controllers' realization on computers must be made. To name just a few TDS discretization methods, state space approaches can be found e.g. in (Engelborghs \& Roose, 2002) based on the so-called solution operator, or in (Breda et al., 2005) via the so-called infinitesimal generator, or using Taylor series expansion of the state vector in a neighborhood of the working point (Hofreiter, 2003). Input-output approaches include e.g. utilization of delta transform operators (Middleton \& Goodwin, 1990; Zítek \& Petrová, 2002). Nevertheless, all these methods consider a "very small" sampling period. An equivalent discrete-time model via the z-transform with a holder ought to be found when the sampling period is "higher". To utilize the z-transform, one has to find a finitedimensional continuous model. This task is the primary aim of this chapter, i.e. to approximate an infinitesimal model of an anisochronic controller such that the final finitedimensional model can be subjected to the z-transform.

In recent decades a huge number of papers and works have been focused on model reduction or rational approximation of TDS, see e.g. (Makilla \& Partington, 1999a, 1999b; Battle \& Miralles, 2000). A fair overview of some methods and approaches has been published in (Partington, 2004). An overwhelming majority of these methods, however, deals with input-output delays only ignoring internal or state delays on the left-hand side of differential equations, i.e. those transfer functions with exponential terms in the denominator. In the contrary, this chapter focuses transfer function rational approximations for TDS with internal (state) delays since there are no theoretical analytic results about approximations convergence and accuracy for such models up today (distinct from single input-output delay which has been deeply analysed by rigorous analytic means many times) and one can thus expect interesting numerical comparative results. The basic question is whether the higher order approximation yields the better (i.e. more accurate) finite dimensional approximating model. The appropriate methods are chosen so that they are easy to deal with and anyone can use them effortlessly. Namely, Padé approximation, shift operator approximations - Laguerre and Kautz shift - and Fourier analysis based method are introduced and benchmarked. The common principle of all approaches consists in substitution of exponential terms in the transfer function of TDS with a rational fraction in the Laplace complex variable $s$.

We made no attempts to deal with analytic mathematical proofs in this chapter. From the practical point of view, the efficiency and accuracy of the selected approaches is measured and compared via norms in the Hardy space, namely, $H_{\infty}$ and $H_{2}$ norms, the definitions of which and some calculation tricks are presented here as well (Štecha \& Havlena, 2000). 
A Matlab-Simulink user-interface application has been programmed to make a benchmark of approximations easier which enables to enter an approximated (nominal) plant transfer function, to select methods to be compared and the choice of norms by which the accuracy of approximation is calculated. It is also possible to specify an order of the approximation for each selected approximation approach. As the outputs, a table of calculated norms and the gain Bode plot (that is relevant to the norms) are displayed. Some study cases on approximations of anisochronic controllers' derived by this algebraic method are introduced and compared in this contribution. For instance, control of an unstable system describing roller skater on a swaying bow (Zítek et al., 2008), or a laboratory heating system (Pekař et al., 2009), are taken as quasi-practical examples.

The chapter is organized as follows. In Section 2 a brief general input-output description of TDS is introduced together with the coprime factorization for the $\mathbf{R}_{M S}$ ring representation. Fundamentals of algebraic controller design in $\mathbf{R}_{M S}$ using a simple control feedback scheme are presented in Section 3. Section 4 introduces selected rational approximation methods for anisochronic controllers' transfer functions. Definitions and calculation issues regarding to norms for approximations' performance comparison are focused in Section 5. The application part of the chapter, i.e. Matlab-Simulink user interface, is described in Section 6. Finally, illustrative benchmark examples are presented in Section 7.

\section{TDS models}

Since the chapter applies finite-dimensional approximations of (controllers') transfer functions, there is no point to introduce TDS systems description in the state space, regardless of the fact that state-space models appear as a result of system modelling and it would be natural to mention them first. For further details about state-space TDS models the reader is referred to (Richard, 2003). The second aim of this section is to present the concept of fields of fractions as an extension of meromorphic description in the form of quasipolynomial fractions, namely the ring of quasipolynomial meromorphic functions, $\mathbf{R}_{M S}$, which is suitable to meet some elementary. So-called retarded TDS are primarily focused; nevertheless, neutral ones have to be mentioned as well, since it is necessary to make the ring definition complete.

\subsection{Input-output quasipolynomial model}

Since the authors' interest lies in single-input single-output (SISO) TDS and their inputoutput models, they are concerned here. Namely, transfer functions in the form of quasipolynomial fractions giving rise to the meromorphic representation are taken as initial models to be approximated.

For both lumped (point-wise) and distributed input-output and internal delays in the system or model, the Laplace transform of a state space model (considering zero initial conditions) can be formulated as follows 


$$
G(s)=\frac{b(s)}{a(s)}
$$

where $b(s), a(s)$ are quasipolynomials of the general form

$$
q(s)=s^{n}+\sum_{i=0}^{n} \sum_{j=1}^{h_{i}} m_{i j} s^{i} \exp \left(-s \vartheta_{i j}\right), \vartheta_{i j} \geq 0
$$

where $n$ is the order of a quasipolynomial which usually agrees with the system degree (of a state-space model), $m_{i j}$ are real numbers and $\vartheta_{i j} \geq 0$ represent delays. If $\sum_{j=1}^{h_{n}} m_{n j} \exp \left(-\vartheta_{n j} s\right)$ does not equal a real constant, the system is called neutral; otherwise, the system is retarded.

\subsection{TDS stability}

A retarded system (1) is said to be asymptotically (exponentially) stable if all poles are located in the open left half plane, $\mathbb{C}_{0}^{-}$, i.e. there is no $s$ satisfying

$$
a(s)=0, \operatorname{Re} s \geq 0, b(s) \neq 0
$$

Condition $b(s) \neq 0$ in (3) is taken into accont if the system contains distributed delays since there hence exist common roots of the transfer function numerator and denominator which are not system poles.

A rather more complicated TDS stability conditions are given regarding to neutral systems, since there may exist vertical strips of system poles tending to the imaginary axis of infinite amplitude. Moreover, these strips can be sensitive to even infinitesimally small deviations in delays, i.e. the position of system poles in the real axis is not continuous with respect to delays. A neutral system (1) is exponentially stable if there is no $s$ such that

$$
a(s)=0, \operatorname{Re} s \geq-\varepsilon, b(s) \neq 0
$$

for arbitrarily $\varepsilon>0$.

A system is strongly stable if so-called associated difference equation (in state-space formulation) remains asymptotically stable when subjected to small variations in delays (Hale \& Verduyn Lunel, 1993), i.e. a TDS remains formally stable, see details in (Byrnes et al., 1984; Loiseau et al., 2002). Formal stability, roughly speaking, means that the rightmost vertical strip of poles does not cross the imaginary axis. If this holds also under small delay changes, the system is strongly stable. A necessary and sufficient strong stability (and thus also formal stability) condition in the Laplace transform can be formulated as

$$
\sum_{j=1}^{h_{n}}\left|m_{n j}\right|<1
$$


according to e.g. (Zítek \& Vyhlídal, 2008), where $m_{n j}$ are real coefficients for the highest $s$ power in the denominator $a(s)$ of (1).

\subsection{TDS model over RMS ring}

Algebraic approaches for analysis and control of TDS can be performed either in the state space or in the realm of input-output models where fields, modules and rings as principal algebraic notions and tools are utilized. Usually, commensurate delays, i.e. those which can be expressed as integer multiples of the smallest one, are assumed; however, delays are naturally real-valued and thus this assumption is rather restrictive for real applications. Non-commensurate or rationally unapproximated delays results in a fraction of quasipolynomials as presented above. However, these transfer function representations are not suitable in order to satisfy some basic control requirements, e.g. controller feasibility, closed-loop (Hurwitz) and formal (strong) stability.

Rather more general approaches (Vidyasagar, 1985; Kučera, 1993) utilize a field of fractions where a transfer function is expressed as a ratio of two coprime elements of a suitable ring. A ring is a set closed for addition and multiplication, with a unit element for addition and multiplication and an inverse element for addition, i.e. division is not generally allowed. A powerful algebraic tool ensuring some basic control requirements, such as internal closedloop stability and controller properness, is a ring of stable and proper RQ-meromorphic functions ( $\left.\mathbf{R}_{M S}\right)$. Since the original definition of $\mathbf{R}_{M S}$ in (Zítek \& Kučera, 2003) does not constitute a ring, some minor changes in the definition were made e.g. in (Pekař \& Prokop, 2009). Namely, although the retarded structure of TDS is considered only, the minimal ring conditions require the use of neutral quasipolynomials at least in the numerator as well. In this chapter, the ring definition is reformulated once more to comprise models of neutral type, distributed delays and formal stability.

A term $T(s)$ from $\mathbf{R}_{M S}$ ring is represented by a proper ratio of two quasipolynomials $y(s) / x(s)$ where a denominator is a quasipolynomial of degree $n$ and a numerator can be factorized as

$$
y(s)=\tilde{y}(s) \exp (-\tau s)
$$

where $\tilde{y}(s)$ is a quasipolynomial of degree $l$ and $\tau \geq 0$. Note that the degree of a quasipolynomial means its highest s-power. The element is analytic and bounded in $\mathbb{C}^{+}$, particularly, there is no pole so such that $\operatorname{Re} s_{0} \geq 0$ (or $\operatorname{Re} s_{0} \geq-\varepsilon, \varepsilon>0$, for neutral terms, more precisely, which can be taken as a generalization) - in other words, all possible roots of $x(s)$ in $\mathbb{C}^{+}$are those of $y(s)$. Thus, it lies in the space $H_{\infty}\left(\mathbb{C}^{+}\right)$providing the finite norm defined as

$$
\|T\|_{\infty}:=\sup \{|T(s)|: \operatorname{Re} s \geq 0\}
$$

It is said that $T(s)$ is $H_{\infty}$ stable (Partington \& Bonnet, 2004). That is, the system has finite $L_{2}(0, \infty)$ to $L_{2}(0, \infty)$ gain where $L_{2}(0, \infty)$ norm of an input or output signal $h(t)$ is defined as 


$$
\|h(t)\|_{2}:=\sqrt{\int_{0}^{\infty}|h(t)|^{2} \mathrm{~d} t}
$$

Notice, for instance, that $T(s)$ having no pole in the right-half complex plane but with a sequence of poles with real part converging to zero can be $H_{\infty}$ unstable due to an unbounded gain at the imaginary axis.

Moreover, $T(s)$ is also formally stable which is guaranteed by condition (5) for $x(s)$. Unfortunately, strong stability can not be included in the ring definition since the product of two strongly stable terms can be strongly unstable. Although neutral TDS that are strongly stable can be $H_{\infty}$ stable, see (Partington \& Bonnet, 2004), yet they are not $H_{\infty}$ (nor BIBO) stabilizable, (Loiseau et al., 2002). It can be shown that neglecting formal stability can bring the problem with ring axioms in controller design, i.e. $H_{\infty}$ stability is not sufficient.

In addition, the ratio is proper, i.e. $l \leq n$. More precisely, there exists a real number $R>0$ for which holds that

$$
\sup _{\operatorname{Re} s>0,|s| \geq R}|T(s)|<\infty
$$

\subsection{Coprime factorization in RMS}

Let the plant be initially described by the transfer function

$$
G(s)=\frac{b(s)}{a(s)}
$$

where $a(s), b(s)$ are quasipolynomials. Hence, using a coprime factorization, a plant model has the form

$$
G(s)=\frac{B(s)}{A(s)}
$$

where $A(s), B(s) \in \mathbf{R}_{M S}$ are coprime, i.e. there does not exist a non-trivial (non-unit) common factor of both elements. Details about divisibility can be analogously deduced from notes presented in (Pekař \& Prokop, 2009). Note that a system of neutral type can induce problem since there can exist a coprime pair $A(s), B(s)$ which is not, however, Bézout coprime - which implies that the system can not be stabilized by any feedback controller admitting the Laplace transform (Loiseau et al., 2002); for instance when the system is not formally stable. More precisely, two coprime elements $A(s), B(s) \in \mathbf{R}_{M S}$ form a Bézout factorization if and only if

$$
\inf _{\operatorname{Re} s \geq 0}(|A(s)|,|B(s)|)>0
$$




\section{Controller design in RMS}

The aim of this section is to outline controller design based on the algebraic approach in the $\mathbf{R}_{M S}$ ring satisfying the closed loop stability in that sense that all transfer functions in the feedback are from the ring (i.e. they lies in $H_{\infty}$ and are proper and formally stable) controller feasibility, reference tracking and load disturbance rejection. As a control system, the simple feedback loop is chosen for the simplicity, see Fig. 1.

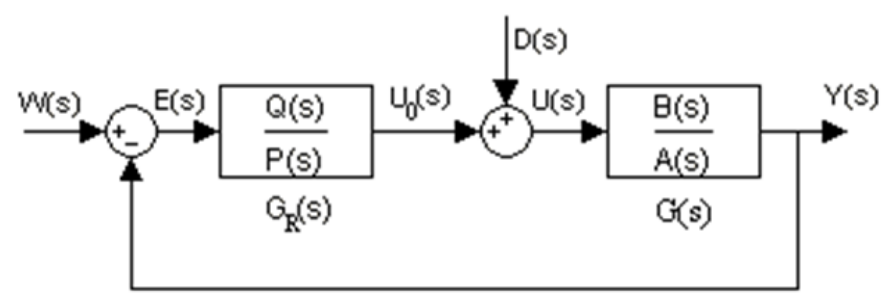

Figure 1. Control feedback scheme

For algebraic controller design in $\mathbf{R}_{M S}$ it is initially supposed that not only the plant is expressed by the transfer function over $\mathbf{R}_{M S}$ but a controller and all system signals are over the ring. Let $W(s)$ be the Laplace transform of the reference signal, $D(s)$ stands for that of the load disturbance, $E(s)$ is transformed control error, $U_{0}(s)$ expresses the controller output (control action), $U(s)$ represents the plant input affected by a load disturbance, and $Y(s)$ is the plant output controlled signal in the Laplace transform. The plant transfer function is depicted as $G(s)$, and $G_{R}(s)$ stands for a controller in the scheme.

External inputs, reference and load disturbance signals, respectively, have forms

$$
W(s)=\frac{H_{W}(s)}{F_{W}(s)}, D(s)=\frac{H_{D}(s)}{F_{D}(s)}
$$

where $H_{W}(s), H_{D}(s), F_{W}(s), F_{D}(s) \in \mathbf{R}_{M S}$.

The following important feedback transfer functions can be derived

$$
\begin{aligned}
& G_{W Y}(s)=\frac{Y(s)}{W(s)}=\frac{B(s) Q(s)}{M(s)}, G_{D Y}(s)=\frac{Y(s)}{D(s)}=\frac{B(s) P(s)}{M(s)} \\
& G_{W E}(s)=\frac{E(s)}{W(s)}=\frac{A(s) P(s)}{M(s)}, G_{D E}(s)=\frac{E(s)}{D(s)}=-\frac{B(s) P(s)}{M(s)}
\end{aligned}
$$

where the controller transfer function is factorized as follows

$$
G_{R}(s)=\frac{Q(s)}{P(s)}
$$

and the common denominator meromorphic function reads 


$$
M(s)=A(s) P(s)+B(s) Q(s)
$$

Meromorphic functions $Q(s), P(s)$ are from $\mathbf{R}_{M S}$ and the fraction (16) is (Bézout) coprime. The numerator of $M(s) \in \mathbf{R}_{M S}$ agrees to the characteristic quasipolynomial of the closed loop generally defined in (2).

A brief description of basic control design steps follows.

\subsection{Closed loop stabilization}

Given a Bézout coprime pair $A(s), B(s) \in \mathbf{R}_{M S}$ the closed-loop system is stable if and only if there exists a pair $P(s), Q(s) \in \mathbf{R}_{M S}$ satisfying the Bézout identity

$$
A(s) P(s)+B(s) Q(s)=1
$$

A particular stabilizing solution of (17), say $P_{0}(s), Q_{0}(s)$, can be then parameterized as

$$
\begin{aligned}
& P(s)=P_{0}(s) \pm B(s) Z(s) \\
& Q(s)=Q_{0}(s) \mp A(s) Z(s)
\end{aligned}
$$

where $Z(s) \in \mathbf{R}_{M S}$. Parameterization (18) is used to satisfy remaining control and performance requirements, such as reference tracking, disturbance rejection etc.

The proof of the statement above can be done analogously as in (Zítek \& Kučera, 2003) where a three-step proof for a similar ring was presented. Condition (12) ensures i.a. that there can exist the ring inversion of $M(s)$ since it proves that there is no common zero of $A(s), B(s)$ in $\mathbb{C}^{+}$(including infinity).

\subsection{Reference tracking}

The task of this subsection is to find $Z(s) \in \mathbf{R}_{M S}$ in (18) so that the reference signal is being tracked. The solution idea results from the form of $G_{W E}(s)$ defined in (14). Consider the limit

$$
\lim _{t \rightarrow \infty} e_{W}(t)=\lim _{s \rightarrow 0} s E_{W}(s)=\lim _{s \rightarrow 0} s G_{W E}(s) W(s)=\lim _{s \rightarrow 0} s A(s) P(s) \frac{H_{W}(s)}{F_{W}(s)}
$$

where ${ }_{W}$ expresses that the signal is a response to the reference not influenced by other external inputs. Limit (19) reaches zero if $\lim _{s \rightarrow 0} E_{W}(s)<\infty$ and $E_{W}(s)$ is analytic and bounded in the right half-plane, i.e. $E_{W}(s) \in H_{\infty}\left(\mathbb{C}^{+}\right)$- this requirement also satisfies that $\lim _{t \rightarrow \infty} \int_{0}^{t} e_{W}(\tau) \mathrm{d} \tau<\infty$. Moreover, it must hold that $G_{W E}(s)$ is proper (or, equivalently, $E_{W}(s)$ is strictly proper) because of the feasibility (impulse free modes) of $e_{W}(t)$. If one 
wants to prevent the closed loop stability from the sensitivity to small delays, or to preserve formal stability at least, the denominator of $E_{W}(s)$ must be a (quasi)polynomial satisfying (5). This implies, in other words, that the reference tracking is fulfilled if $E_{W}(s) \in \mathbf{R}_{M S}$.

Alternatively, $F_{W}(s)$ must divide the product $A(s) P(s)$ in $\mathbf{R}_{M S}$. Hence, all unstable zeros (including infinity) of $F_{W}(s)$ must be those of $A(s) P(s)$ and, moreover, the quasipolynomial numerator of $F_{W}(s)$ is formally (strongly) stable. It means that one has to set all unstable zeros of $F_{W}(s)$ (with corresponding multiplicities) as zeros of $P(s)$ - if there is no one already contained in $B(s)$. Recall that zeros mean zero points of a whole term in $\mathbf{R}_{M S}$, not only those of a quasipolynomial numerator.

Note that the controller approach fails for formally unstable controlled processes since then the feedback loop remains formally unstable (neutral term can not be affected by a controller).

\subsection{Load disturbance rejection}

The attenuation of the load disturbance signal entering a plant model can be done analogously as for reference tracking. Thus, $Z(s)$ is chosen so that $Y_{D}(s) \in \mathbf{R}_{M S}$ which is clear from

$$
\lim _{t \rightarrow \infty} y_{D}(t)=\lim _{s \rightarrow 0} s Y_{D}(s)=\lim _{s \rightarrow 0} s G_{D Y}(s) D(s)=\lim _{s \rightarrow 0} s B(s) P(s) \frac{H_{D}(s)}{F_{D}(s)}
$$

where ${ }_{D}$ means that the output is influenced only by the disturbance. Or, $F_{D}(s)$ must divide the product $B(s) P(s)$ in $\mathbf{R}_{M S}$.

One has to be careful when deciding about the form of $Z(s)$ since both divisibility conditions must be fulfilled simultaneously. A detailed procedure of reference tracking and disturbance rejection briefly described above was presented e.g. in (Pekař \& Prokop, 2011).

\section{Selected transfer function rational approximations}

Selected easy-handling transfer function rational approximation methods based on the substitution of exponential elements by a rational function are described in this section. The emphasis is put on the engineering usability of the methods.

\subsection{Padé approximation}

In the second half of the $19^{\text {th }}$ century, a French mathematician Henry Padé devised a simple and, nowadays, one of the most used and favorite rational approximations which is based on the comparison of derivatives of the approximating and approximated functions in zero. More precisely, let $F(s)$ with $F(0) \neq 0$ be analytic in the neighborhood of zero. Then, the $n$ - 
$n$ Padé approximation is the function $\Phi(s)=N_{n}(s) / D_{n}(s)$ where $N_{n}(s), D_{n}(s)$ are polynomials of the $n$th order with $D_{n}(0)=1$ and it holds that $F^{(i)}(0)=\Phi^{(i)}(0), i=1, \ldots, 2 n$.

Padé approximation of $F(s)=\exp (-s T), T \geq 0$ is given by the following relation (Partington, 2004)

$$
\begin{aligned}
& \exp (-s T) \approx \frac{P(-s)}{P(s)} \\
& P(s)=\sum_{k=0}^{n}\left(\begin{array}{l}
n \\
k
\end{array}\right) \frac{(2 n-k) !}{(2 n) !}(s T)^{k}
\end{aligned}
$$

where $n$ is the order of the approximation. Obviously, one can approximate another function, e.g. the whole transfer function.

A method called diagonal Padé approximation, which is distinguished by some authors, see (Battle \& Miralles, 2000; Richard, 2003) can be expressed as

$$
\begin{aligned}
& \exp (-s T) \approx \frac{P(s)}{P(-s)} \\
& P(s)=\sum_{k=0}^{n} \frac{(2 n-k) !}{k !(n-k) !}(-s T)^{k}
\end{aligned}
$$

However, it is easy to verify, that (21) and (22) represent the same approximations. In fact,

$$
\left(\begin{array}{l}
n \\
k
\end{array}\right) \frac{(2 n-k) !}{(2 n) !}=\frac{n !}{(2 n) ! k !(n-k) !}
$$

and $n ! /(2 n)$ ! is the common factor of both, the approximating numerator and denominator, hence, the fractions are the same.

\subsection{Shift operator approximations}

Approaches based on operator shifting yields from the fact that a delay term $\exp (-s T)$ can be perceived as a shift operator and can be subjected to Maclaurin series expansion. Moreover, the variable $s$ can be vied as a derivative operator.

Hence

$$
\begin{aligned}
& s f(t):=f^{\prime}(t) \\
& \exp (-s T) f(t):=f(t-T)=f(t)-T f^{\prime}(t)+\frac{T^{2}}{2} f^{\prime \prime}(t)-\frac{T^{3}}{6} f^{\prime \prime \prime}(t)+\ldots \\
& \exp (-s T) f(t):=\exp \left(-T \frac{\mathrm{d}}{\mathrm{d} t}\right) f(t)=\left(1-T \frac{\mathrm{d}}{\mathrm{d} t}+\frac{T^{2}}{2} \frac{\mathrm{d}^{2}}{\mathrm{~d} t^{2}}-\frac{T^{3}}{6} \frac{\mathrm{d}^{3}}{\mathrm{~d} t^{3}}+\ldots\right) f(t)
\end{aligned}
$$


A concise overview of some important shift operator approaches follows.

\subsubsection{Laguerre shift}

The eventual relation for this shift operator approximant is given by the formula

$$
\exp (-s T) \approx\left(\frac{1-\frac{s T}{2 n}}{1+\frac{s T}{2 n}}\right)^{n}
$$

where $n$ is the order of the approximation again, see (Makilla \& Partington, 1999a) for more details. The Laguerre shift is successfully used in robust control.

\subsubsection{Kautz shift}

As presented in (Makilla \& Partington, 1999a), the $n$th order Kautz shift reads

$$
\exp (-s T) \approx\left(\frac{1-\frac{s T}{2 n}+\frac{1}{2}\left(\frac{s T}{2 n}\right)^{2}}{1+\frac{s T}{2 n}+\frac{1}{2}\left(\frac{s T}{2 n}\right)^{2}}\right)^{n}
$$

In the source referenced above, there has been analytically proved for input-output delays that the Kautz shift approximation is asymptotically twice more accurate than the Laguerre one.

\subsubsection{Padé shift}

This type of shift approximation is based on the second order Padé approximation (Makilla \& Partington, 1999b; Battle \& Miralles, 2000) and it is also called Padé-2. It can be formulated e.g. as follows

$$
\exp (-s T) \approx\left(\frac{1-\frac{s T}{2 n}+\frac{1}{3}\left(\frac{s T}{2 n}\right)^{2}}{1+\frac{s T}{2 n}+\frac{1}{3}\left(\frac{s T}{2 n}\right)^{2}}\right)^{n}
$$

\subsubsection{Fourier analysis based method}

This approximation methodology results from the analysis of the delay-in-feedback step response or, generally, the response to the input of the form $u(t)=t^{k}, t, k \geq 0$. It has been found that the feedback system response is a superposition of a periodic and an aperiodic 
signal. The Fourier series expansion of the periodic part of the response gives the resulting approximation.

To demonstrate the initial idea more precisely, consider a single delay term $\exp (-s T)$ in the simple negative feedback loop. Then the whole feedback transfer function $W(s)$ reads

$$
W(s)=\frac{Y(s)}{U(s)}=\frac{\exp (-s T)}{1+\exp (-s T)}
$$

Hence

$$
y(t)=-y(t-T)+u(t-T)
$$

and the output $y(t)$ is assumed to be a superposition of a polynomial $q(t)$ and a periodic part $p(t)$

$$
y(t)=q(t)+p(t)
$$

Inserting (30) into (29) yields

$$
y(t-T)-y(t)=\text { constant }
$$

From the Fourier series expansion of $p(t)$ and some further nontrivial calculations, the following final relation can be obtained

$$
W(s)=\frac{1}{2}-\frac{1}{4} T s+\frac{2}{\pi^{2}} T^{3} s^{3} \sum_{k=0}^{n} \frac{1}{(2 n+1)^{2}} \frac{1}{T^{2} s^{2}+(2 n+1) \pi}
$$

The exponential term can be then approximated by the inverse of (28) as

$$
\exp (-s T)=\frac{W(s)}{1-W(s)}
$$

The reader is referred to (Battle \& Miralles, 2000) for more details about the derivation and methodology.

\section{Approximation performance evaluation using norms}

The accuracy of rational approximation methods and approaches has been usually measured and evaluated via conscientious and rigorous mathematic analytic proofs. The derivation of such results has been facilitated by the fact that delays in input-output relation only, ignoring the effect of internal delays, have been considered. This is the main gap which ought to be healed up; however, no analytic methods are used in this chapter anyway. 
Transfer function norms in the Hardy space known from robust control for unstructured uncertainty measurement instead of any complex analytic method for the comparison of approximation accuracy are used in this contribution since this conception is sufficient for particular cases and for engineering practice. Moreover, analytic derivations of accuracies for internal delays would be much more difficult than those for input-output ones.

First of all, define the objective to be minimized in various meanings. The difference between the nominal $G$ and approximated $\hat{G}$ transfer functions is taken as a measured expression $G_{M}$, i.e.

$$
G_{M}=G-\hat{G}
$$

We chose two the most used norms in the Hardy space, namely, $H_{2}$ and $H_{\infty}$ norms, which are effective to express the proximity of transfer functions in the frequency domain. The definitions and some basic properties are then followed by some calculation tricks when dealing with the norms.

\section{1. $\mathrm{H}_{2}$ norm}

The $\mathrm{H}_{2}$, sometimes called quadratic, norm of a stable strictly proper transfer function is defined as

$$
\|G\|_{2}=\frac{1}{\sqrt{2 \pi}} \sqrt{\int_{-\infty}^{\infty}|G(\mathrm{j} \omega)|^{2} \mathrm{~d} \omega}
$$

The norm is finite for strictly proper stable systems having no pole on the imaginary axis, and the meaning of $\mathrm{H}_{2}$ is energy of $G$. A generalized strict properness can be expresses as follows

$$
\lim _{R \rightarrow \infty} \sup _{\operatorname{Re} s>0,|s| \geq R}|G(s)|=0
$$

Note that for TDS with distributed delays, there can exist a denominator root (or roots) of $G$ which is not the system pole.

In computer practice, i.e. working with discrete samples, the integral in (36) is calculated as a sum within a finite range of nonnegative frequencies, $\omega \in\left[0, \omega_{\max }\right]$. Due to the symmetry, the resulting value is doubled finally. The value of $\omega_{\max }$ can be chosen so that the frequency gain is "small enough".

Residual expansion can be used when analytic (and continuous) calculation of $\|G\|_{2}$, (Štecha \& Havlena, 2000). It holds that

$$
\|G\|_{2}=\sqrt{\sum_{\operatorname{Re}_{i}<0} \operatorname{res}_{s_{i}} G(s) G(-s)}
$$


where $s_{i}$ are (stable) system poles and resuduum of a complex function $F(s)$ reads

$$
\underset{s_{i}}{\operatorname{res}} F(s)=\frac{1}{(m-1) !} \lim _{s \rightarrow s_{i}}\left\{\frac{\mathrm{d}^{(m-1)}}{\mathrm{d} s^{(m-1)}}\left[F(s)\left(s-s_{i}\right)^{m}\right]\right\}
$$

where $m$ is the multiplicity of a pole.

\section{2. $\mathrm{H}_{\infty}$ norm}

The H-infinity $\left(H_{\infty}\right)$ norm is defined as

$$
\|G\|_{\infty}=\sup _{\omega}|G(\mathrm{j} \omega)|
$$

i.e. it expresses the supreme of the amplitude (gain) frequency characteristics of $G$, see Fig. 2. If the system is asymptotically (exponentially) stable and provides a finite $H_{\infty}$ norm, it is said that it is $H_{\infty}$ stable and lies in the space $H_{\infty}\left({ }^{+}\right)$of functions analytic and bounded in the right-half complex plane.

The norm is also called $L_{2}$ gain, That is, the $H_{\infty}$ stable system has finite $L_{2}(0, \infty)$ to $L_{2}(0, \infty)$ gain where $L_{2}(0, \infty)$ norm of an input or output signal $h(t)$ is defined as

$$
\|h(t)\|_{2}:=\sqrt{\int_{0}^{\infty}|h(t)|^{2} \mathrm{~d} t}
$$

The frequency characteristics supreme can be easily found by standard analytic means, or by mapping the values of $G(\mathrm{j} \omega)$ when using digital computers.

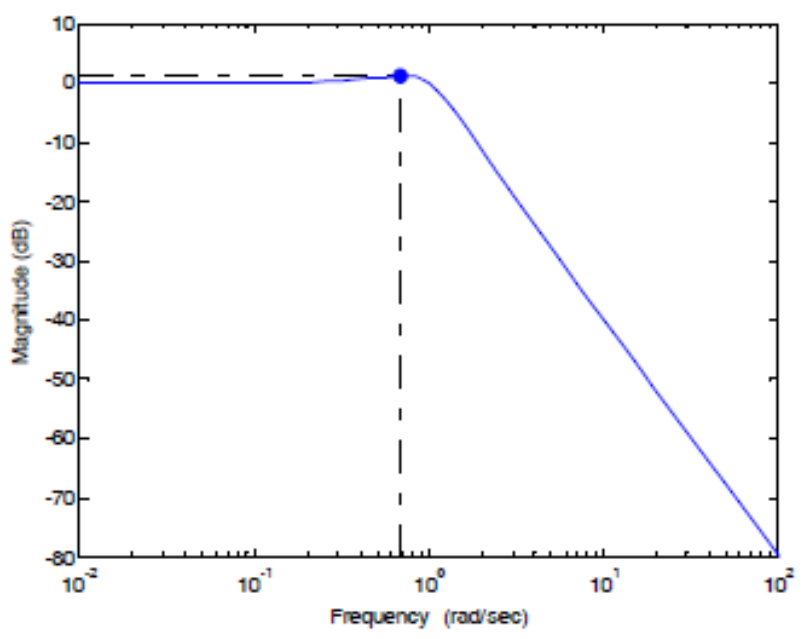

Figure 2. The meaning of $H_{\infty}$ norm as the frequency characteristics supreme 
Note, for instance, that a transfer function having no pole on the imaginary axis but a sequence of poles with real part converging to zero can have an infinite $H_{\infty}$ norm due to an unbounded gain, see (Partington \& Bonnet, 2004).

\section{Matlab-Simulink user interface}

The main application part of this chapter consists in development of a simulation program that can evaluate the quality of approximations. A user-friendly interface in MatlabSimulink environment has been hence developed by the authors in order to provide testing and comparison of approximation approaches introduced above. The source code can be found in (Pekař \& Kurečková, 2011).

Fig. 3 displays the working environment of the application. In the upper part of the user interface, one is allowed to enter a nominal plant transfer function as a numerator and denominator quasipolynomials. The user must be careful since the syntax scheme which is indicated by grey font (or by the help hint) in the edit box must be kept.

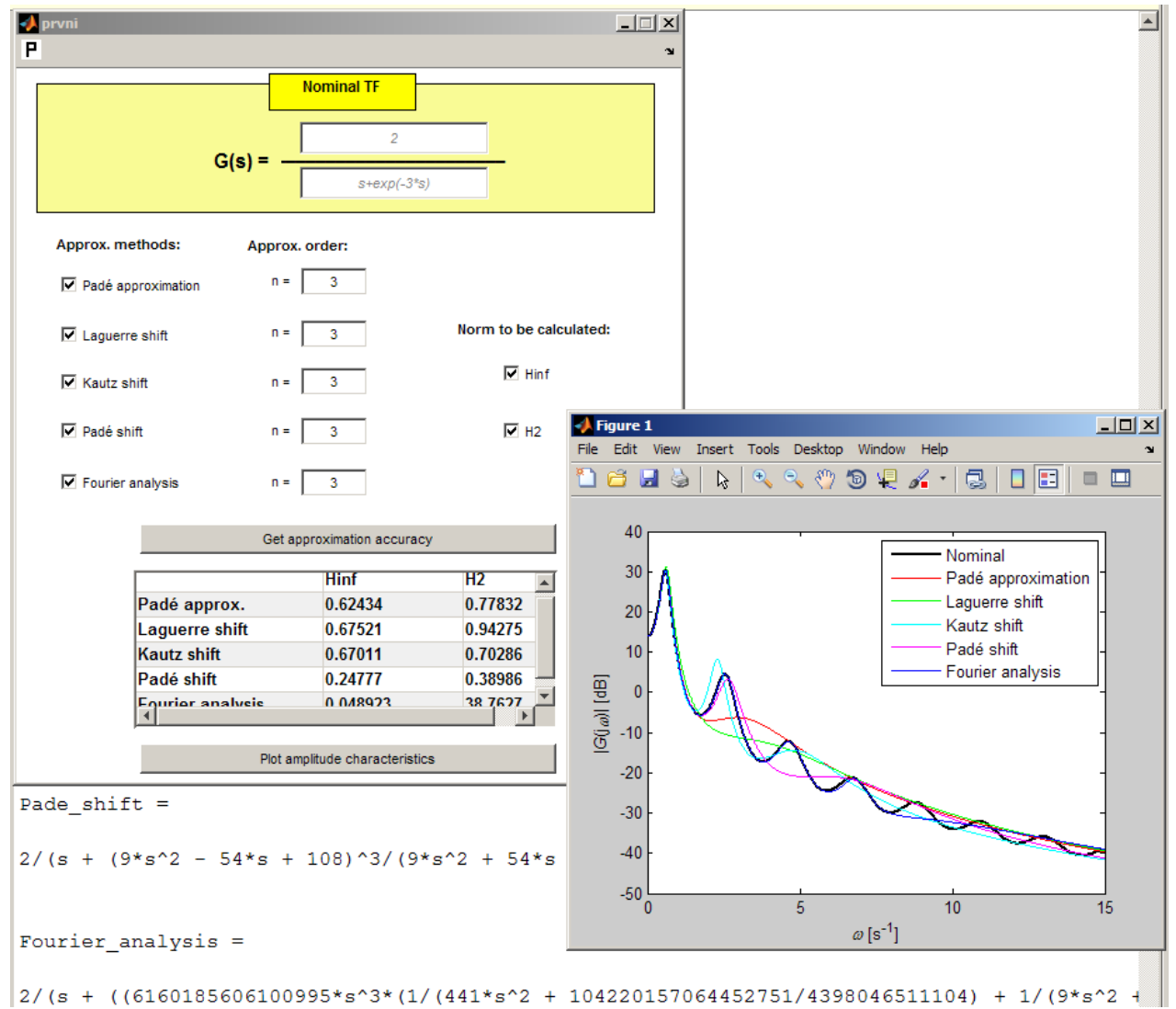

Figure 3. Matlab-Simulink user interface and working environment 
Then, a user can select one or more approximation methods to be compared and check the choice of norms by which the accuracy of approximation is calculated. It is also possible to specify an order of the approximation for each selected method.

By clicking the button Get approximation accuracy, the programme returns a table of calculated norms in the text form. A graphical comparison of amplitude frequency responses for all chosen approximations is obtained by clicking the button Plot amplitude characteristics.

Final approximation controller transfer functions are returned to the Matlab workspace to the command line.

\section{Benchmark examples}

The aim of this section is to verify introduced approximation methods using the user interface. The approximation accuracy with respect to the order of an approximation is a very attractive question as well. As will be seen from the following examples, some rather surprising results can be obtained.

As mentioned above, results of the benchmark ought to be primarily used by the authors for the rational approximation of controllers with internal delays (also called anisochronic) as a first step of controllers' discretization via the z-transform, for the computer implementation.

Three examples of algebraic controller design with rational approximations using the user interface follow. The first one gives results for anisochronic controllers design in control of a hypothetic simple stable LTI with stepwise reference, to demonstrate mainly the design procedure. The second one presents the approximation of an anisochronic controller for an attractive unstable system of a skater on a swaying bow. The third example deals with control of a laboratory circuit heating system, as a typical real-life representative of retarded TDS.

\subsection{Stable system with stepwise reference}

Let the plant be described by the transfer function of a stable first order TDS model with both internal and input-output delays as

$$
G(s)=\frac{b \exp (-\tau s)}{s+a \exp \left(-\vartheta_{s}\right)}, \tau, \vartheta \geq 0, a \vartheta \in(0,2 \pi)
$$

Condition $a \vartheta \in(0,2 \pi)$ ensures the asymptotic stability of the model.

Coprime (Bézout) factorization is the first step of controller design in $\mathbf{R}_{M S}$ ring as follows

$$
G(s) \frac{\frac{b \exp (-\tau s)}{m(s)}}{\frac{s+a \exp (-\vartheta s)}{m(s)}}=\frac{B(s)}{A(s)}, A(s), B(s) \in \mathbf{R}_{M S}
$$


where $m(s)$ is an appropriate stable (quasi)polynomial of the first order (due to the coprimeness). A suitable form of $m(s)$ is contentious and depends on user's requirements, let $m(s)=s+m_{0}, m_{0}>0$.

Consider the simplest practical case that both external inputs are from the class of step functions, hence

$$
W(s)=\frac{H_{W}(s)}{F_{W}(s)}=\frac{\frac{w_{0}}{m_{w}(s)}}{\frac{s}{m_{w}(s)}}, D(s)=\frac{H_{D}(s)}{F_{D}(s)}=\frac{\frac{d_{0}}{m_{d}(s)}}{\frac{s}{m_{d}(s)}}
$$

where $m_{w}(s)$ and $m_{d}(s)$ are arbitrary stable (quasi)polynomials of degree one, say, for the simplicity, $s+m_{0}$ again, $H_{W}(s), F_{W}(s), H_{D}(s), F_{D}(s) \in \mathbf{R}_{M S}$ and $w_{0}$ and $d_{0}$ are real constants. Find a stabilizing particular solution by (17). Set e.g. $Q_{0}=1$ which yields

$$
P_{0}(s)=\frac{s+m_{0}-b \exp (-\tau s)}{s+a \exp (-\vartheta s)}
$$

Now parameterize the solution according to (18) to obtain controllers asymptotically rejecting the load disturbance and tracking the stepwise reference

$$
P(s)=\frac{s+m_{0}-b \exp (-\tau s)}{s+a \exp (-\vartheta s)}-\frac{b \exp (-\tau s)}{s+m_{0}} Z(s)
$$

The numerator of $P(s)$ has to have at least one zero root. Moreover, it is appropriate to have $P(s)$ in a simple form, which is fulfilled e.g. when

$$
Z(s)=\left(\frac{m_{0}}{b}-1\right) \frac{s+m_{0}}{s+a \exp \left(-\vartheta_{s}\right)}
$$

providing

$$
P(s)=\frac{s+m_{0}(1-\exp (-\tau s))}{s+a \exp (-\vartheta s)}, Q=\frac{m_{0}}{b}
$$

Thus, final controller's structure is the following

$$
G_{R}(s)=\frac{m_{0}\left[s+a \exp \left(-\vartheta_{s}\right)\right]}{b\left[s+m_{0}(1-\exp (-\tau s))\right]}
$$

The obtained control structure can be easily compared with the well-known Smith predictor structure and note that the controller is of the anisochronic type because of delay in the 
transfer function denominator. It is naturally possible to take $m(s)$ as a quasipolynomial instead of polynomial; however, this option would make a controller more complicated. The importance of $m(s)$ reveals from the closed loop transfer function

$$
G_{W Y}(s)=\frac{Y(s)}{W(s)}=\frac{m_{0} \exp (-\tau s)}{s+m_{0}}
$$

i.e. $m(s)$ appears as a characteristic (quasi)polynomial of the closed loop.

Model (41) can to fit the dynamics of a high order undelayed system; for instance, a tenth order system governed by the transfer function

$$
G_{s}(s)=\frac{1}{(2 s+1)^{10}}
$$

can be estimated by model (41) with $a=b=6.5 \cdot 10^{-2}, \tau=15.3, \vartheta=6.7$, see (Zítek \& Vyhlídal, 2003; Pekař \& Prokop, 2008) for details. Hence, let the system has these parameters and $m_{0}=0.5$.

Let $n=\{1,2,3,4,5\}$, where $n$ is the order of the approximation method, and test the accuracies for all methods introduced above. The best results for each of them measured by $H_{\infty}$ and $H_{2}$ norms with the corresponding order are displayed in Table 1.

Obviously, the best result for $H_{\infty}$ is given alongside by the Padé approximation and Laguerre shift of the first order, whereas, amazingly, higher orders make results worse. The Fourier analysis based methods yields almost the same score for all studied orders. The benchmark results for the $H_{2}$ norm with $\omega_{\max }=15$ introduced in Table 1 are almost identical with those for $H_{\infty}$, i.e. the Padé approximation and Laguerre shift of the first order are the best and the Fourier analysis based methods gives almost the same results for all orders. The corresponding gain frequency responses for the approximations of orders as in the last column in Table 1 are displayed in Fig. 4.

\begin{tabular}{|l|l|l|l|l|}
\hline Method & $H_{\infty}$ & $n$ & $H_{2}$ & $n$ \\
\hline Padé approx. & 0.243 & 1 & 0.237 & 1 \\
\hline Laguerre shift & 0.243 & 1 & 0.237 & 1 \\
\hline Kautz shift & 0.408 & 5 & 0.299 & 3 \\
\hline Padé shift & 0.277 & 1 & 0.29 & 1 \\
\hline Fourier analysis & 0.38 & 1 & 0.354 & 5 \\
\hline
\end{tabular}

Table 1. Comparison of rational approximations of (49) measured by $\mathrm{H}_{\infty}$ and $\mathrm{H}_{2}$ norms

The approximating transfer function by Padé approximation and Laguerre shift with $n=1$ is given by (51), which agrees with a conventional PID controller 


$$
\hat{G}_{R}(s)=\frac{0.7692 s^{2}+0.506 s+0.0065}{s^{2}+0.2307 s}
$$

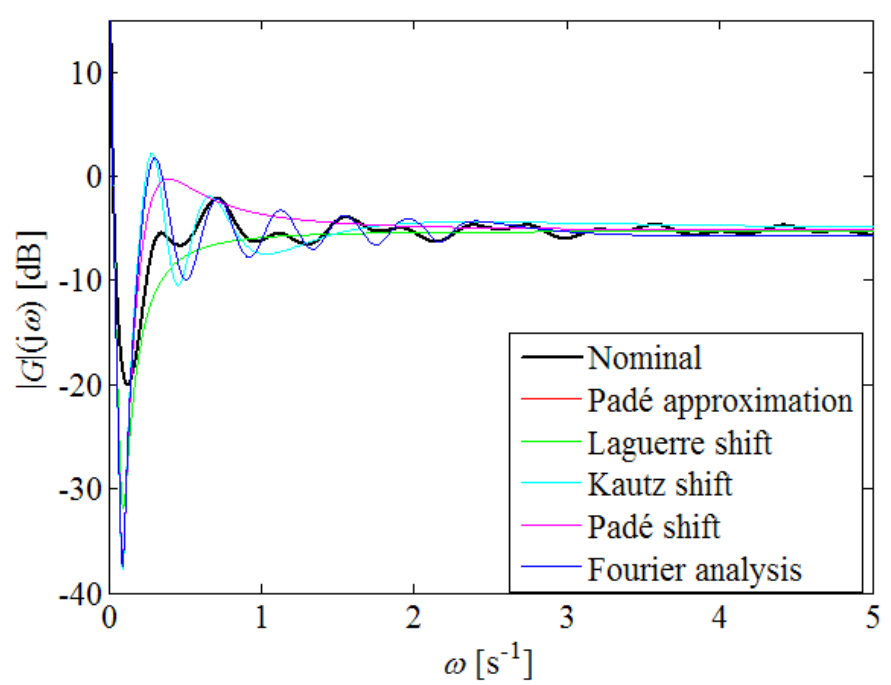

Figure 4. Bode magnitude plots of nominal and approximating models for (48)

\subsection{Control of the roller skater on a swaying bow}

Consider an unstable system describing roller skater on a swaying bow, (Zítek et al., 2008), governed by the transfer function

$$
G(s)=\frac{Y(s)}{U(s)}=\frac{b \exp (-(\tau+\vartheta) s)}{s^{2}\left(s^{2}-a \exp (-\vartheta s)\right)}
$$

see Fig. 5, where $y(t)$ is the skater's deviation from the desired position, $u(t)$ expresses the slope angle of a bow caused by force $P$, delays $\tau, \vartheta$ means the skater's and servo latencies, respectively, and $b, a$ are real parameters. Skater controls the servo driving by remote signals into servo electronics.

Let the model parameters be $b=0.2, a=1, \tau=0.3 \mathrm{~s}, \vartheta=0.1 \mathrm{~s}$, as in the literature, and design the controller structure analogously as in the previous subsection 7.1. Consider that the reference and load disturbance are in the form of step-wise functions. Then the final controller has the structure given by the following transfer function

$$
G_{R}(s)=\frac{b\left(q_{3} s^{3}+q_{2} s^{2}+q_{1} s+q_{0}\right)\left(s+m_{0}\right)^{4}+p_{0} m_{0}^{4} s^{2}\left(s^{2}-a \exp (-\vartheta s)\right)}{b\left[\left(s^{3}+p_{2} s^{2}+p_{1} s+p_{0}\right)\left(s+m_{0}\right)^{4}-p_{0} m_{0}^{4} \exp (-(\tau+\vartheta) s)\right]}
$$


where $p_{2}, p_{1}, p_{0}, q_{3}, q_{2}, q_{1}, q_{0} \in \circ$ are free parameters, see details in (Pekař \& Prokop, 2011b). Using a quasi-optimal tuning algorithm, the parameters were set as

$$
q_{3}=4.7587, q_{2}=2.1164, q_{1}=2.6252, q_{0}=0.4482, p_{2}=0.4636, p_{1}=0.529, p_{0}=4.6164
$$

and e.g. $m_{0}=5$.

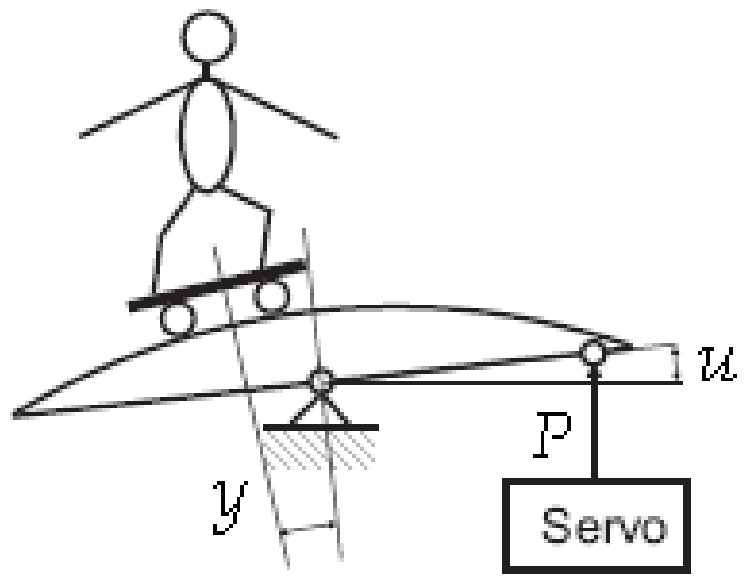

Figure 5. The roller skater on a swaying bow

The comparison of the best controller rational approximations can be found in Table 2 . Again, the method based on the Fourier series expansion very slowly approaches the limit value of the $H_{2}$ norm $(\approx 7.811)$ with the increasing $n$. The only method evincing the better asymptotical results with the higher order approximation is the Kautz shift. Again, the Padé and Laguerre approximations of the first order give very good results with the approximating controller transfer function

$$
\begin{aligned}
& 4.7587 s^{8}+121.084 s^{7}+1245.21 s^{6}+20969.8 s^{5}+90315.2 s^{4}+39304.5 s^{3} \\
\hat{G}_{R}(s)= & \frac{+56753.5 s^{2}+9604.38 s+1400.63}{s^{8}+25.4636 s^{7}+262.119 s^{6}+1383.74 s^{5}+3952.16 s^{4}+6389.1 s^{3}+8872.37 s^{2}} \\
& +18964.6 s
\end{aligned}
$$

\begin{tabular}{|l|l|l|l|l|}
\hline Method & $H_{\infty}$ & $n$ & $H_{2}$ & $n$ \\
\hline Padé approx. & 5.6674 & 1 & 8.577 & 1 \\
\hline Laguerre shift & 5.6674 & 1 & 8.577 & 1 \\
\hline Kautz shift & 6.0847 & 5 & 9.7446 & 5 \\
\hline Padé shift & 6.0668 & 1 & 9.674 & 1 \\
\hline Fourier analysis & 5.6674 & 1 & 7.8114 & 5 \\
\hline
\end{tabular}

Table 2. Comparison of rational approximations of (53) measured by $H_{\infty}$ and $H_{2}$ norms 
Fig. 6 displays Bode magnitude plots for the best orders for $\mathrm{H}_{2}$ (the last column in Table 2), which verifies a very good performance of all the approaches.

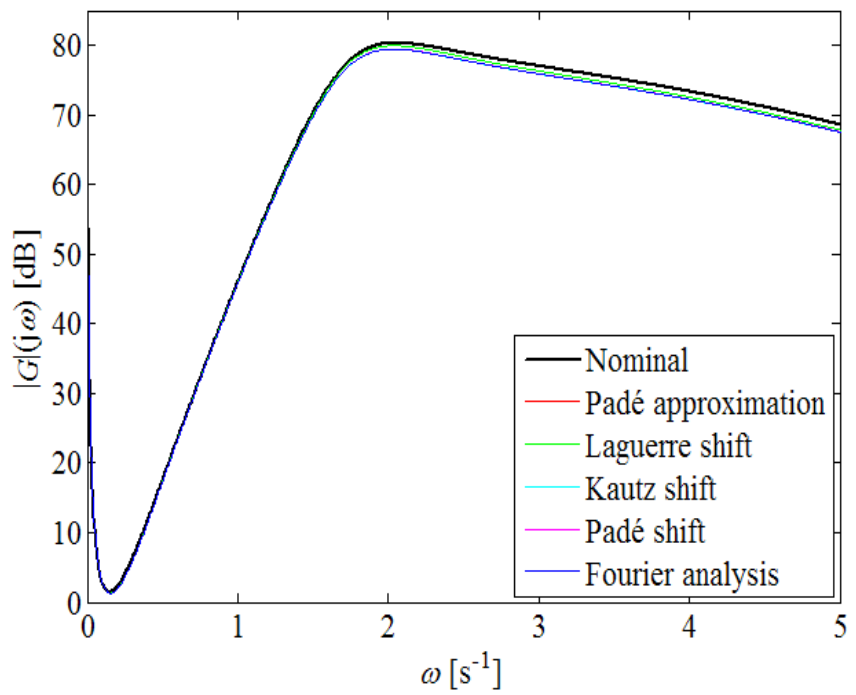

Figure 6. Bode magnitude plots of nominal and approximating models for (53)

\subsection{Control of a circuit heating plant}

The laboratory heating plant, a photo and a sketch of which, respectively, are displayed in Fig. 7, was assembled at the Faculty of Applied Informatics of Tomas Bata University in Zlín in order to test control algorithms for systems with dead time. The original description of the apparatus and its electronic circuits can be found in (Dostálek et al., 2008).
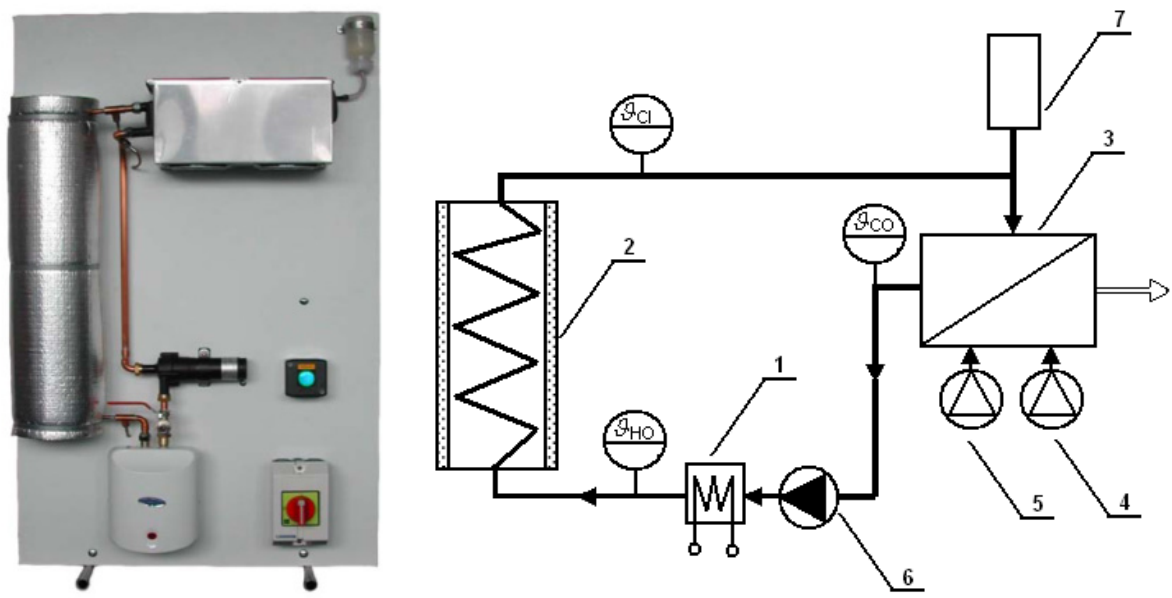

Figure 7. Bode magnitude plots of nominal and approximating models for (53) 
The heat transferring fluid (namely distilled water) is transported using a continuously controllable DC pump $\{6\}$ into a flow heater $\{1\}$ with maximum power $P(t)$ of $750 \mathrm{~W}$. The temperature of a fluid at the heater output is measured by a platinum thermometer giving value of $\vartheta_{H O}(t)$. Warmed liquid then goes through a 15 meters long insulated coiled pipeline $\{2\}$ which causes the significant delay in the system. The air-water heat exchanger (cooler) $\{3\}$ with two cooling fans $\{4,5\}$ represents a heat-consuming appliance. The speed of the first fan can be continuously adjusted, whereas the second one is of on/off type. Input and output temperatures of the cooler are measured again by platinum thermometers giving $\vartheta_{C I}(t)$, resp. $\vartheta_{C O}(t)$. The expansion tank $\{7\}$ compensates for the expansion effect of the water.

Originally, it was intended to control input delays only; however, it was shown that the plant contains internal delays as well. A detailed mathematical model was presented in (Pekař et al., 2009). A linearized model of the relation between the power to the heater $P(t)$ and $\vartheta_{\mathrm{CO}}(t)$ can be expressed by the transfer function

$$
G(s)=\frac{\vartheta_{C O}(s)}{P(s)}=\frac{\left[b_{0 D} \exp \left(-\tau_{0} s\right)+b_{0}\right] \exp (-\tau s)}{s^{3}+a_{2} s^{2}+a_{1} s+a_{0}+a_{0 D} \exp \left(-\vartheta_{s}\right)}
$$

where all real parameters in the model are complex algebraic functions of physical quantities in the circuit and input and output steady states, see details in the literature. It was determined that for a certain working point, the parameters are

$$
\begin{aligned}
& b_{0 D}=2.334 \cdot 10^{-6}, b_{0}=-2.146 \cdot 10^{-7}, a_{2}=0.1767, a_{1}=0.009, a_{0}=1.413 \cdot 10^{-4}, \\
& a_{0 D}=-7.624 \cdot 10^{-5}, \tau_{0}=1.5, \tau=131, \vartheta=143
\end{aligned}
$$

The controller structure obtained by controller design in $\mathbf{R}_{M S}$ yields

$$
G_{R}(s)=\frac{P(s)}{\vartheta_{C O}(s)}=\frac{m_{0}^{3}\left(s^{3}+a_{2} s^{2}+a_{1} s+a_{0}+a_{0 D} \exp \left(-\vartheta_{s}\right)\right)}{b_{0}\left(s+m_{0}\right)^{3}-m_{0}^{3}\left[b_{0 D} \exp \left(-\tau_{0} s\right)+b_{0}\right] \exp (-\tau s)}
$$

Table 3 displays the best values for approximations at their orders $n=\{1,2,3,4,5\} . H_{2}$ norm is measured within the frequency range $\omega \in[0.001 ; 0.5]$ with the discretization step $\Delta \omega=0.001$.

\begin{tabular}{|l|l|l|l|l|}
\hline Method & $H_{\infty}$ & $n$ & $H_{2}$ & $n$ \\
\hline Padé approx. & 27994.7 & 4 & 1197.3 & 5 \\
\hline Laguerre shift & 28528.6 & 3 & 1194.3 & 2 \\
\hline Kautz shift & 28365.9 & 2 & 1196.4 & 2 \\
\hline Padé shift & 28650.2 & 3 & 1198.7 & 1 \\
\hline Fourier analysis & 28760.9 & 1 & 1205.1 & 1 \\
\hline
\end{tabular}

Table 3. Comparison of rational approximations of (56) measured by $H_{\infty}$ and $H_{2}$ norms 
Immense values of $H_{\infty}$ norm are caused by a pair controller poles which is very close to the imaginary axis $\left(s_{i}=0.0002 \pm 0.094 \mathrm{j}\right)$ and the controller is obviously unstable. Padé approximation of the $4^{\text {th }}$ order provides the best value for $H_{\infty}$, whereas Laguerre shift of the $2^{\text {nd }}$ order gives the best approximation measured by $\mathrm{H}_{2}$ norm. Again, this example indicates that the higher order of the approximation does not mean the more accurate result automatically.

The comparison of Bode magnitude plots for the best orders (which are placed in the last column in Table 3) for $\mathrm{H}_{2}$ can be seen in Fig. 8.

To conclude study cases above, it is startling that the best approximations measured by $\mathrm{H}_{2}$ and $H_{\infty}$ norms are mostly given by the well known and widely used Padé approximation of the first (or a low) order. By simulations, the higher order of an approximation does not generally yields the more accuracy finite dimensional model, which is in the contradiction with a general expectation and analytic results for rational approximations for TDS with input-outputs delays. Comparative Bode plots above indicate the usability and a very good efficiency of selected methods.

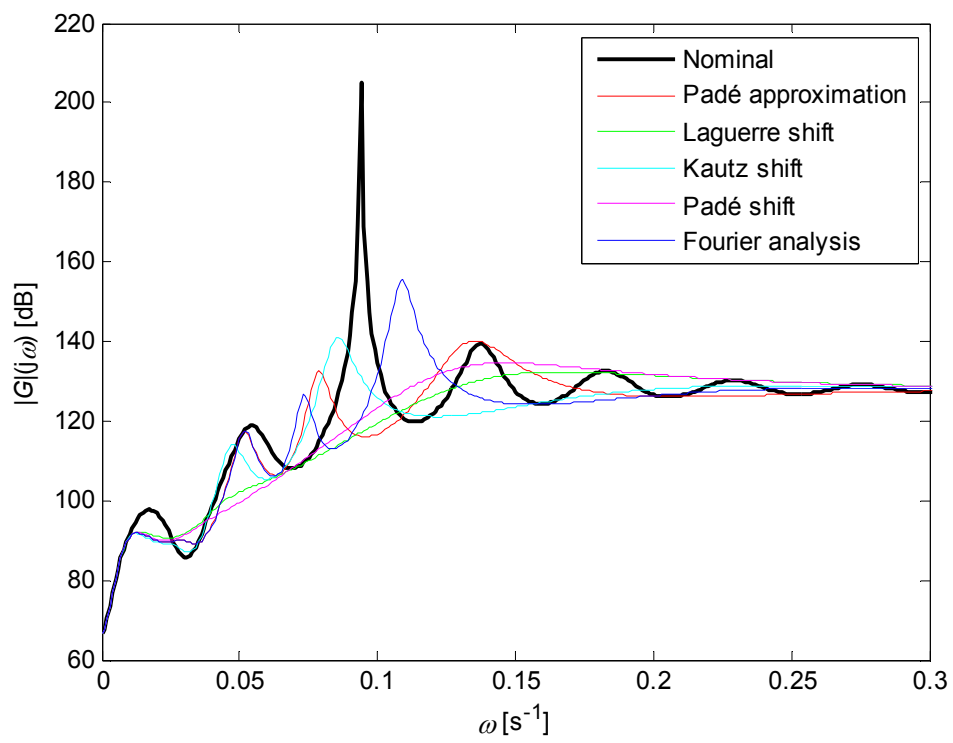

Figure 8. Bode magnitude plots of nominal and approximating models for (58).

\section{Conclusion}

This chapter intended to propound the reader a methodology for algebraic controller design for systems with internal delays, followed by a comparison of several easy-handling techniques for rational (i.e. finite-dimensional) approximation of anisochronic (i.e. infinitedimensional) controllers - or their transfer functions, more precisely. 
The first, controller design, part was based on the solution of the Bézout identity, to obtain stable and proper controllers, along with the Youla-Kučera parameterization for reference tracking and load disturbance rejection, in $\mathbf{R}_{M S}$ ring.

The second, controller approximation, part of this chapter intended to use five quite simple methods, namely Padé approximation, Laguerre, Kautz shift and Fourier analysis based method, how to approximate a delay exponential term in the transfer function. Two norms in the Hardy space, namely, $H_{2}$ and $H_{\infty}$ norms were used as a measure for the accuracy and efficiency of the approaches since there are no rigorous analytic results about the approximation efficiency and accuracy in the literature and these norms are usually sufficient in technical practice. Approximation methods were selected so that they are serviceable also for people with basic mathematical background knowledge. The authors plan to use approximated controllers in their digital implementation on PC or PLC.

Matlab with Simulink was a very useful assistant here. The authors programmed a simple user interface which enables the user to enter a nominal transfer function and select approximation methods to be used and their orders. As a result, the program returns the accuracies in both text and graphical forms.

Simulation experiments with the program were made. Control of a simple stable TDS, control of unstable TDS of a skater on the swaying bow and control of a laboratory circuits heating plant were benchmark examples. The results were very interesting and startling because the habitual Padé approximation proved to be very good and, moreover, the higher order approximation did not automatically mean the better result for systems with internal delays.

\section{Author details}

Libor Pekař, Eva Kurečková and Roman Prokop

Tomas Bata University in Zlín, Czech Republic

\section{Acknowledgement}

The authors kindly appreciate the financial support which was provided by the Ministry of Education, Youth and Sports of the Czech Republic, under the project CEBIA-Tech No. CZ.1.05/2.1.00/03.0089.

\section{References}

Battle, C. \& Miralles, A. (2000). On the Approximation of Delay Elements by Feedback. Automatica, Vol.36, No.5, pp. 659-664, ISSN 0005-1098

Bellman, R. \& Cooke, K.L. (1963). Differential-Difference Equations, Academic Press, New York Brethé, D. \& Loiseau, J.J. (1998). An Effective Algorithm for Finite Spectrum Assignment of Single-Input Systems with Delays. Mathematics and Computers in Simulation, Vol.45, No.3-4, pp. 339-348, ISSN 0378-4754 
Byrnes, C.I., Spong, M.W. \& Tarn, T.J. (1984). A Several Complex Variables Approach to Feedback Stabilization of Neutral Delay-Differential Systems. Mathematical Systems Theory, Vol.17, No.1, pp. 97-133, ISSN 0025-5661

Conte, G. \& Perdon, A.M. (2000). Systems over Rings: Geometric Theory and Applications. Annual Reviews in Control, Vol.24, pp. 113-124, 1367-5788

Dostálek, P., Dolinay, J. \& Vašek, V. (2008). Design and Implementation of Portable Data Acquisition Unit in Process Control and Supervision Applications. WSEAS Transactions on Systems and Control, Vol.3, No.9, pp. 779-788, ISSN 1991-8763

El'sgol'ts, L.E. \& Norkin, S.B. (1973). Introduction to the Theory and Application of Differential Equations with Deviated Arguments. Academic Press, New York

Findensen, W., Pulaczewski, J. \& Manitius, A. (1970). Multilevel Optimization and Dynamic Coordination of Mass Flows in a Beet Sugar Plant. Automatica, Vol.6, No.4, 1970, pp. 581-589, ISSN 0005-1098

Gluesing-Lueerssen, H. (1997). A Behavioral Approach to Delay-Differential Systems. SIAM Journal of Control and Optimization, Vol.35, pp. 480-499, ISSN 0363-0129

Górecki, H.; Fuksa, S.; Grabowski, P. \& Korytowski, A. (1989). Analysis and Synthesis of Time Delay Systems, John Wiley \& Sons, ISBN 978-047-1276-22-7, New York

Hale, J.K., \& Verduyn Lunel, S.M. (1993). Introduction to Functional Differential Equations. In: Applied Mathematical Sciences, Vol.99, Springer-Verlag, ISBN 978-038-7940-76-2, New York

Štecha, J., Havlena, V. (2000). Modern Control Theory (in Czech). ČVUT Publishing, ISBN 8001-01971-3, Prague

Kolmanovskii, V.B. \& Nosov, V.R. (1986). Stability of Functional Differential Equations, Academic Press, ISBN 0124179401, London

Kolmanovskii, V.B. \& Myshkis, A. (1999). Introduction to the Theory and Applications of Functional Differential Equations, Cluwer Academy, ISBN 978-0792355045, Dordrecht, Netherlands

Krasovskii, N.N. (1963). Stability of Motion, Standford University Press, Chicago

Kučera V. (1993). Diophantine Equations in Control - A Survey. Automatica, Vol.29, No.6, pp. 1361-1375, ISSN 0005-1098

Loiseau, J.J., Cardelli, M. \& Dusser, X. (2002). Neutral-Type Time-Delay Systems that are not Formally Stable are not BIBO Stabilizable. IMA Journal of Mathematical Control and Information, Vol.19, No.1-2, pp. 217-227, ISSN 0265-0754

Makilla, P.M. \& Partington, J.R. (1999a). Laguerre and Kautz Shift Approximations of Delay Systems. International Journal of Control, Vol.72, No.10, pp. 932-946, ISSN 0020-7179

Makilla, P.M. \& Partington, J.R. (1999b). Shift Operator Induced Approximations of Delay Systems. SIAM Journal of Control and Optimization, Vol. 37, No.6, pp. 1897-1912, ISSN 0363-0129

Manitius, A.Z. \& Olbrot, A.W. (1979). Finite Spectrum Assignment Problem for Systems with Delays. IEEE Transactions on Automatic Control, Vol.24, No.4, pp. 541-553, ISSN 0018-9286 
Morávka, J. \& Michálek, K. (2008). Anisochronous Model of the Metallurgical RH Process. Transactions of the VŠB - Technical University of Ostrava, Mechanical Series, Vol.14, No.2, 2008, pp. 91-96, ISSN 1210-0471

Niculescu, S.-I. (2001). Delay Effects on Stability. In: Lecture Notes in Control and Information Sciences, Vol.269, Springer, ISBN 9781852332914, Berlin

Partington, J.R. (2004). Some Frequency-Domain Approaches to the Model Reduction of Delay Systems. Annual Reviews in Control, Vol.28, No.1, pp. 65-73, ISSN 1367-5788

Partington, J.R. \& Bonnet, C. (2004). $H_{\infty}$ and BIBO Stabilization of Delay Systems of Neutral Type. Systems \& Control Letters, Vol.52, No.3-4, 2004, pp. 283-288, ISSN 0167-6911

Pekař, L. \& Kurečková, E. (20/03/2011). Application for Transfer Function Rational Approximations for Time Delay Systems, In: Faculty of Applied Informatics, Tomas Bata University in Zlín, 22/03/2011, Available from:

http://web.fai.utb.cz/?id=0_2_2_5\&iid=0\&lang=cs\&type $=0$

Pekař, L. \& Prokop R (2008). An approach for relay based identification of anisochronic models, Proceedings of the 27th IASTED International Conference on Modelling, Identification and Control, paper No. 596-061 [CD-ROM], ISBN 978-0-88986-712-3, Innsbruck, Austria, February 11-13, 2008

Pekař, L. \& Prokop R (2009). Some Observations about the RMS Ring for Delayed Systems, Proceedings of the 17th International Conference on Process Control '09, pp. 28-36, ISBN 97880-227-3081-5, Štrbské Pleso, Slovakia, June 9-12, 2009

Pekař, L. \& Prokop, R. (2010). Control Design for Stable Systems with Both Input-Output and Internal Delays by Algebraic Means, Proceedings of the 29th International Conference on Modelling, Identification and Control, pp. 400-407, ISBN 978-0-88986-833-5, Innsbruck, Austria, February 15-17, 2010

Pekař, L. \& Prokop, R. (2011a). On Reference Tracking and Disturbance Rejection for Time Delay Systems, Proceedings of the 31st International Conference on Modelling, Identification and Control, pp. 327-333, ISBN 978-088986863-2, Innsbruck, Austria, February 14-16, 2011

Pekař, L. \& Prokop, R. (2011b). Implementation of a New Quasi-Optimal Controller Tuning Algorithm for Time-Delay Systems, In: MATLAB for Engineers - Applications in Control, Electrical Engineering, IT and Robotics, K. Perůtka, (Ed.), pp. 3-26. InTech, ISBN 978-953307-914-1, Rijeka, Croatia

Pekař, L., Prokop, R. \& Dostálek, P. (2009). Circuit Heating Plant Model with Internal Delays. WSEAS Transactions on Systems, Vol.8, No.9, pp. 1093-1104, ISSN 1109-2777

Pekař, L., Prokop, R. \& Dostálek, P. (2011). Non-Stepwise Reference Tracking for Time Delay Systems, Proceedings of the 12th International Carpathian Control Conference, pp. 292-297, ISBN 978-1-61284-360-5, Velké Karlovice, Czech Republic, May 25-28, 2011

Richard, J.P. (2003). Time-delay Systems: an Overview of Some Recent Advances and Open Problems. Automatica, Vol.39, No.10, pp. 1667-1694, ISSN 0005-1098

Simuenovic, G. (2011). Separate Identification of Coefficients and Delays in Time-Delay Systems, Ph.D. Thesis, Faculty of Mechanical Engineering, Czech Technical University in Prague

Vidyasagar, M. (1985). Control System Synthesis: A Factorization Approach, MIT Press, ISBN 978-0262220279, Cambridge, M. A. 
Volterra, V. (1928). Sur le Théorie Mathématique des Phénomenès Héreditaires. Journal des Mathématiques Pures et Appliquées, Vol.7, 1928, pp. 249-298

Vyhlídal, T. \& Zítek, P. (2001). Control System Design Based on a Universal First Order Model with Time Delays. Acta Polytechnica, Vol.44, No.4-5, 2001, pp. 49-53, ISSN 1210-2709

Zítek, P. (1983). Anisochronic State Theory of Dynamic Systems. Acta Technica ČSAV, Vol.4, 1983, ISSN 0001-7043

Zítek, P. (1997). Frequency Domain Synthesis of Hereditary Control Systems via Anisochronic State Space. International Journal of Control, Vol.66, No.4, pp. 539-556, ISSN 0020-7179

Zítek, P. \& Hlava, J. (2001). Anisochronic Internal Model Control of Time-Delay Systems. Control Engineering Practice, Vol.9, No.5, pp. 501-516, ISSN 0967-0661

Zítek, P. \& Kučera, V. (2003). Algebraic Design of Anisochronic Controllers for Time Delay Systems. International Journal of Control, Vol.76, No.16, pp. 1654-1665, ISSN 0020-7179

Zítek, P.; Kučera, V. \& Vyhlídal, T. (2008). Meromorphic Observer-based Pole Assignment in Time Delay Systems. Kybernetika, Vol.44, No.5, pp. 633-648, ISSN 0023-5954

Zítek, P. \& Víteček, A. (1999). Control of Systems with Delays and Nonlinearities (in Czech), ČVUT Publishing, ISBN 80-01-01939-X, Prague

Zítek, P. \& Vyhlídal, T. (2003). Low Order Time Delay Approximation of Conventional Linear Model, Proceedings of 4th IMACS Symposium on Mathematical Modelling, 2003, pp. 28, ISBN 3-901608-24-9, Vienna, Austria, February 5-7, 2003

Zítek, P. \& Vyhlídal, T. (2008). Argument-Increment Based Stability Criterion for Neutral Time Delay Systems, Proceedings of the 16th Mediterranean Conference on Control and Automation, pp. 824-829, ISBN 978-1-4244-2505-1, Ajaccio, Corsica, France, June 25-27, 2008 